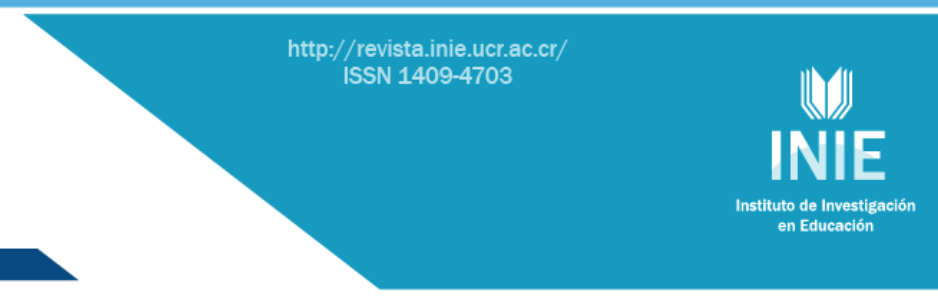

\title{
CONSTRUCCIÓN DE UNA COMUNIDAD VIRTUAL DE APRENDIZAJE AMBIENTAL (CVAA): ESPACIO INTERACTIVO PARA AMBIENTALIZAR EL QUEHACER UNIVERSITARIO ESTATAL DE COSTA RICA
}

BUILDING A VIRTUAL COMMUNITY FOR ENVIRONMENTAL LEARNING (VCEL): AN INTERACTIVE SPACE TO ENHANCE ENVIRONMENTALISM INTO THE REGULAR OBLIGATIONS OF PUBLIC UNIVERSITIES IN COSTA RICA

Volumen 11, Número Especial pp. 1-23

Este número se publicó el 30 de junio de 2011

\author{
Claudia Zúñiga Vega \\ Jacqueline García Fallas \\ Elizabeth Arnáez Serrano \\ Lidia Hernández Rojas \\ María de los Ángeles Carrillo Delgado \\ Claudia Charpentier Esquivel \\ Patricia Rojas Núñez \\ Sonia Arguedas Quirós
}

La revista está indexada en los directorios:

LATINDEX, REDALYC, IRESIE, CLASE, DIALNET, DOAJ, E-REVIST@S,

La revista está incluida en los sitios:

REDIE, RINACE, OEI, MAESTROTECA, PREAL, HUASCARAN, CLASCO 


\title{
CONSTRUCCIÓN DE UNA COMUNIDAD VIRTUAL DE APRENDIZAJE AMBIENTAL (CVAA): ESPACIO INTERACTIVO PARA AMBIENTALIZAR EL QUEHACER UNIVERSITARIO ESTATAL DE COSTA RICA \\ BUILDING A VIRTUAL COMMUNITY FOR ENVIRONMENTAL LEARNING (VCEL): AN INTERACTIVE SPACE TO ENHANCE ENVIRONMENTALISM INTO THE REGULAR OBLIGATIONS OF PUBLIC UNIVERSITIES IN COSTA RICA
}

\author{
Claudia Zúñiga Vega ${ }^{1}$ \\ Jacqueline García Fallas ${ }^{2}$ \\ Elizabeth Arnáez Serrano ${ }^{3}$ \\ Lidia Hernández Rojas ${ }^{4}$ \\ María de los Ángeles Carrillo Delgado \\ Claudia Charpentier Esquivel ${ }^{6}$ \\ Patricia Rojas Núñez \\ Sonia Arguedas Quirós ${ }^{8}$
}

Resumen: Este artículo tiene como objetivo presentar el caso de la construcción y puesta en práctica de la Comunidad Virtual de Aprendizaje Ambiental (CVAA) en Costa Rica, cuya página está disponible en la siguiente dirección www.comunidad.inie.ucr.ac.cr. Es un sitio Web que servirá para dar un mayor impulso a la incorporación del eje ambiental en la vida universitaria costarricense, así como para hacer un recuento de sus actividades hasta el 2011. La CVAA ha facilitado el intercambio académico para recuperar, analizar, discutir, compartir y gestar experiencias que faciliten la incorporación de la dimensión ambiental en el quehacer cotidiano de las universidades estatales. Se concluye que esta estrategia tecnológica favorece la participación de diferentes sectores universitarios, la transversalización de la dimensión ambiental y el desarrollo profesional en dicho ámbito.

Palabras clave: COMUNIDADES VIRTUALES, DOCENCIA UNIVERSITARIA, DIMENSIÓN AMBIENTAL, COMUNIDAD VIRTUAL DE APRENDIZAJE AMBIENTAL

\begin{abstract}
The purpose of this article is to present a case study related to the building and practicality of a virtual community for environmental learning (VCEL, www.comunidad.inie.ucr.ac.cr) in Costa Rica. This website will serve as the driving force to the integration of a curricula into the environmental education in the Costa Rican university life. The paper also shows the virtual community activities throughout the year 2011. The VCEL (CVAA, acronyms in Spanish) has made the academic exchange possible in order to retrieve, analyze, compare and develop experiences that facilitate the integration of an environmental dimension into the everyday duties of public universities. The conclusions of this article determine that this technological strategy favors the involvement of all of the participants at the university level (faculty, students and administration), encourages the mainstreaming of the environmental dimension and promotes professional development in the environmental field.
\end{abstract}

Key words: VIRTUAL COMMUNITIES, TEACHING AT THE UNIVERSITY LEVEL, ENVIRONMENTAL DIMENSION, VIRTUAL COMMUNITY FOR ENVIRONMENTAL LEARNING

\footnotetext{
${ }^{1}$ Dirección electrónica: czuniga@itcr.ac.cr

2 Dirección electrónica: jgarciafallas@gmail.com

3 Dirección electrónica: earnaez@itcr.ac.cr

${ }^{4}$ Dirección electrónica: Imhernandez@uned.ac.cr

${ }^{5}$ Dirección electrónica: idespo@gmail.com

6 Dirección electrónica: ccharpen@gmail.com

7 Dirección electrónica: arojasn@gmail.com

8 Dirección electrónica: sargued@una.ac.cr
}

Artículo recibido: 11 de mayo, 2011

Aprobado: 16 de iunio, 2011 


\section{CONTEXTO DE LA INVESTIGACIÓN}

Las universidades estatales costarricenses han definido políticas acordes con las necesidades y demandas de la sociedad, que requieren incluir como un eje transversal al componente ambiente. La Subcomisión Interuniversitaria de Educación Ambiental (CIEA) perteneciente al Consejo Nacional de Rectores (CONARE) de Costa Rica ha realizado diferentes esfuerzos para facilitar al profesorado de las universidades estatales, la construcción de estrategias y espacios educativos que promuevan una cultura ambiental. La CIEA en sus más de 15 años de creada

...ha desarrollado diferentes proyectos de investigación y formación continua, con el fin de estimular su compromiso con los temas y acciones ambientales, así como con la identificación de estrategias didácticas para incorporarlas en la formación de profesionales de diferentes ciencias y disciplinas, involucrando todos los sectores sociales: administrativo, académico y estudiantil. (Hernández y García, 2009, p. 3)

En consecuencia como parte de la práctica multiplicadora para incluir la Dimensión Ambiental (DA) se propuso la construcción y el desarrollo de una comunidad de aprendizaje virtual para ambientar el quehacer universitario por medio de la educación ambiental, en el periodo 2007- 2009. Su objetivo consistía en promover, en la comunidad universitaria, la construcción de conocimientos, habilidades y destrezas, que permitan la incorporación de la Dimensión Ambiental (DA) en su quehacer". De esta manera, se implementaría el uso de las tecnologías de la información y de la comunicación (TIC) para atender las necesidades de formación en este campo.

Al respecto, la Comunidad Virtual de Aprendizaje Ambiental (CVAA), busca cubrir las necesidades docentes para vincular al estudiantado con una nueva cultura ambiental, que incida en el desarrollo de la sociedad costarricense. Y a la vez, es un ejemplo de cómo se integran la "sociedad de la información y del conocimiento" en un contexto universitario.

Por tal motivo, la CVAA se construyó tomando como base el estudio de los referentes teóricos y conceptuales de las tecnologías de la información y la comunicación (TIC), el tecnoconstructivismo, la Educación Ambiental y la Dimensión Ambiental. 
El enfoque metodológico de esta investigación es afín a la orientación propuesta por Elliott (1996, p. 66), según la cual

el movimiento de investigación-acción en educación constituye una señal de la tendencia a la transformación existente en la cultura universitaria de los encargados de la formación profesional de las instituciones de enseñanza superior, como de la transformación de la cultura profesional de los docentes.

Este enfoque facilitó el acompañamiento reflexivo tanto en los procesos de construcción y su puesta práctica, como en la realización de sesiones del equipo investigador y, al mismo tiempo, con diferentes grupos universitarios.

El compromiso de las personas involucradas en dichos procesos se reflejó en el desarrollo de acciones tales como: la participación en sesiones presenciales y virtuales, la elaboración de propuestas para incorporar la DA en su quehacer y la retroalimentación de la experiencia, entre otros.

Al asumirse la investigación-acción como la metodología a seguir en este proceso, se opta por el modelo de Lewin, interpretado por Kemmis (citado por Elliott, 1996, p. 89), que implica el desarrollo de una idea inicial (la propuesta por el equipo de investigadoras), el reconocimiento de la situación y su relación con la propuesta inicial e implica el desarrollo de la CVAA, su implementación, revisión y evaluación constante en colaboración con las personas involucradas en el proceso.

\section{Fundamentos pedagógicos de la Comunidad Virtual De Aprendizaje}

La CVAA se fundamentó en las siguientes líneas conceptuales, las cuales en su conjunto establecen la visión pedagógica de los procesos que se propiciaron, a saber:

- Educación Ambiental

- Dimensión Ambiental

- Comunidad de aprendizaje virtual

- Tecno-constructivismo

- Comunicación ambiental 


\section{Educación Ambiental (EA)}

Se entiende como un proceso que reconoce valores, aclara conceptos y fomenta las destrezas y las actitudes necesarias para comprender y apreciar las interrelaciones entre el ser humano, su cultura y su medio biofísico; entraña también la práctica en la toma de decisiones y en la propia elaboración de un código de comportamiento con respecto de las situaciones relacionadas con la calidad del entorno inmediato del ciudadano (UNESCO-UICN, 1970 citado por Curiel, 1997, p. 6).

La educación ambiental busca promover en las personas el desarrollo de una actitud favorable hacia la acción ambiental positiva responsable. Sobre la base de la teoría expuesta por Emmons (1997), se define como el comportamiento que el ser humano adquiere hacia el medio, producto de la integración del conocimiento, valores, sensibilidad y actitudes positivas, que se concreta en habilidades y procedimientos personales que reflejan el convencimiento de la pertenencia del ser humano al ambiente. Una acción ambiental positiva es una conducta producto del empoderamiento y del sentimiento de pertenencia al ambiente.

Es preciso concentrarse en fomentar el desarrollo de valores ambientales en busca de la transformación de una cultura respetuosa hacia el ambiente. Un valor es una preferencia fuerte y duradera que un individuo o un grupo tiene para un objeto, conducta o modo de vida (Rokeach citado por Caduto, 1985). Las creencias y actitudes que se posean van a influir en los valores que las personas asumen, éstos afectan tanto la forma de vida de los seres humanos como el entorno, es decir, el ambiente en general.

El compromiso que se asume con el planeta tiene como fin conservarlo, por esta razón, los valores se deben poner en práctica para lograr ese objetivo y así aprender a detectar las causas de los problemas ambientales para prevenirlos o mitigarlos.

La CIEA concibe la EA como un proceso, que involucra diversas etapas o pasos que incluyen el desarrollo de: una conciencia ambiental, actitudes favorables con el ambiente, aptitudes para el análisis de los problemas ambientales, la capacidad de evaluación de la realidad ambiental y la capacidad de participación en la resolución y prevención de problemas ambientales. Para promover la EA como proceso se presentan a continuación algunas acciones para su abordaje (Cuadro 1).

Por lo anterior, en la CVAA se considera a la Educación Ambiental (EA) como una Dimensión para permear todo el currículo con un enfoque de transversalidad, con el propósito 
de propiciar valores y conductas, que permitan abordar positivamente la relación con el ambiente.

\section{CUADRO 1}

Promoción de la Educación Ambiental (EA) como un proceso

\begin{tabular}{l}
\hline PASOS \\
\hline $\begin{array}{l}\text { Desarrollo de una conciencia } \\
\text { ambiental }\end{array}$
\end{tabular}
ACCIONES

Analizar causas y efectos de los problemas ambientales.

Adoptar modos de vida compatibles con la conservación de la calidad del medio.

Percibir la importancia del ambiente en las actividades de desarrollo económico, social y cultural.

Apropiación de conocimientos sobre aspectos ambientales.

Construcción de información pertinente sobre la situación ambiental.

Transferencia de conocimientos a situaciones reales.

Búsqueda de alternativas o desarrollo de estrategias, para tomar acciones frente a los problemas ambientales próximos.

Desarrollo de actitudes favorables El desarrollo de autoconciencia y autoconcepto. con el ambiente

El desarrollo de un juicio moral (justicia, equidad, dignidad personal). La adquisición de capacidades para la argumentación y el diálogo.

Desarrollo de aptitudes para el
análisis de los problemas
ambientales

Desarrollo de la capacidad de evaluación de la realidad ambiental
Brindar herramientas (habilidades) para que el individuo pueda:

- Participar en un proceso de investigación.

- Evaluar el entorno

- Tomar decisiones sobre los problemas del entorno con el propósito de que los pueda identificar y resolver.

Obtener datos de interés para comprender mejor los problemas del entorno.

Descubrir el impacto de las acciones desarrolladas.

Tomar decisiones pertinentes para buscar soluciones concretas o líneas de acción.

La participación es el fin último de la educación ambiental, por ello, los individuos deben ser capaces de elegir, tomar decisiones y ser consecuentes con las propias elecciones. participación en la resolución y prevención de problemas ambientales

Fuente: Elaboración propia 


\section{Dimensión Ambiental (DA)}

Se concibe como un eje transversal articulador del plan de estudios, cuya función es impregnar todo el currículo con un enfoque que propicia valores y conductas en el estudiantado, el cual le permite abordar constructivamente su relación con el medio que le rodea. Representa una articulación conceptual, metodológica y de significación para la formación.

Constituye un punto de encuentro entre las ciencias naturales y las ciencias sociales, es un importante eje de articulación interdisciplinario que proporciona una mayor congruencia de la realidad, en que las ideas y los hechos relacionados con el binomio ser humano-ambiente adquieren nuevos sentidos.

Para hacer una realidad este eje articulador, es necesario innovar en cuanto a lo conceptual, lo metodológico y lo disciplinario, en busca de la creación de proyectos integrales que reestructuren radicalmente el currículo; de esta manera, se logra una transformación efectiva de la cultura ambiental construida por los profesores y sus estudiantes. Esta orientación conceptual sobre la DA ha sido elaborada a partir de los aportes de González Gaudiano (1993) y Novo (1993).

La dinámica para la incorporación de la DA en el quehacer universitario, cuando se trabaja en la CVAA, se representa en la Figura No. 1. En el centro del círculo se introduce a los participantes y las facilitadoras. En esta comunidad, la comunicación y la difusión de los resultados son fundamentales. Por ello, para el trabajo de la misma, se dispone de módulos educativos, experiencias universitarias y población universitaria sensibilizada. A la vez, puede generar nuevas experiencias universitarias, materiales educativos y relaciones inter $e$ intrauniversitarias que permitirán desarrollar proyectos y otras actividades, que promuevan una nueva cultura ambiental. 
FIGURA No. 1

Comunidad Virtual de Aprendizaje Ambiental por las universidades estatales costarricenses para incluir la Dimensión Ambiental en el quehacer académico

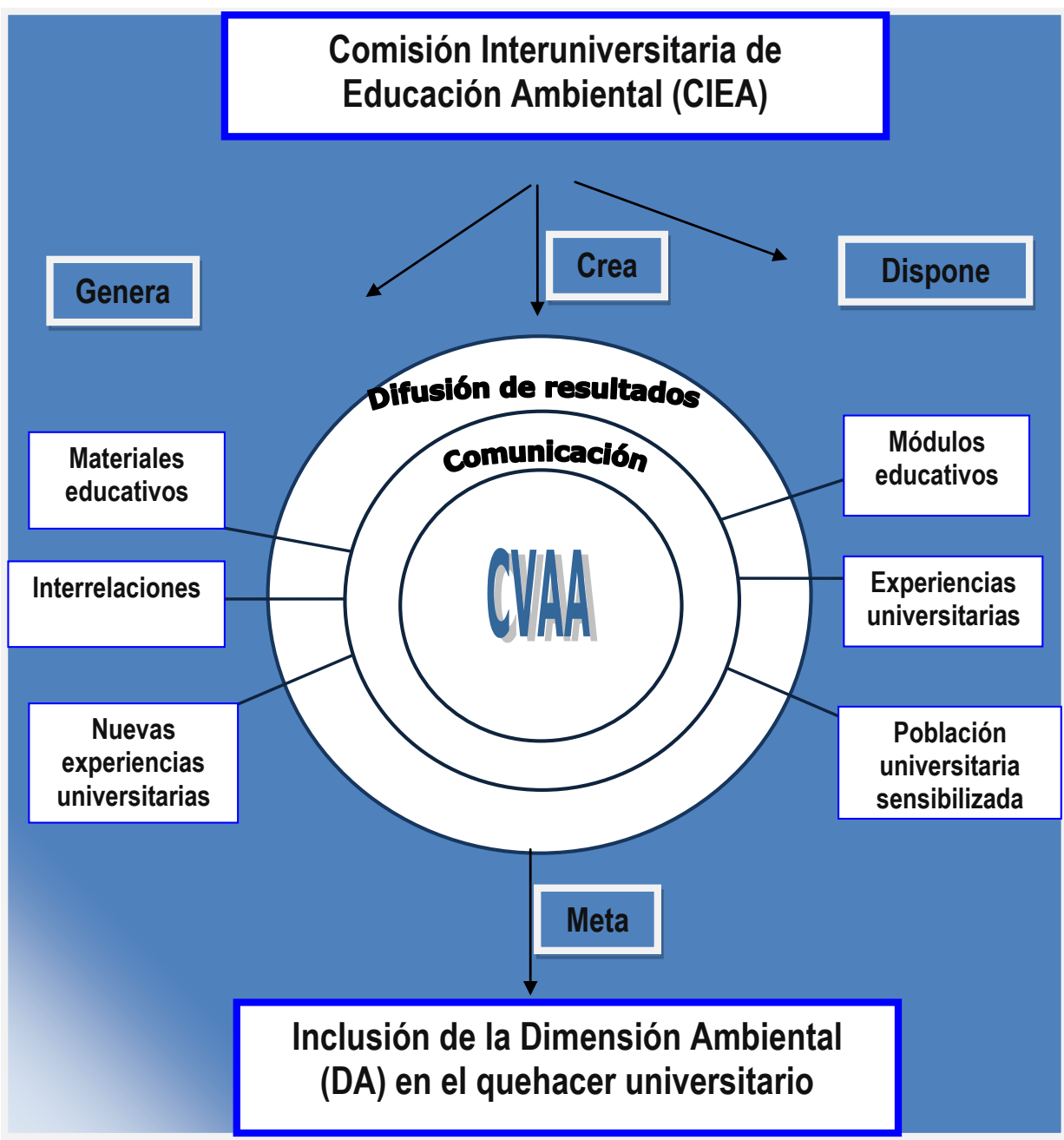

Fuente: Elaboración propia

Además, la CVAA brinda espacios para el trabajo interuniversitario y para el intercambio de experiencias, no solo de estudiantes sino también del personal docente y administrativo, ya que estos últimos también deben llegar a tener aprendizaje ambiental como parte del sistema universitario. 


\section{Tecno-constructivismo}

Es una aproximación teórica que integra las TIC con la visión pedagógica constructivista, puesto que estas tecnologías permiten ampliar las posibilidades para compartir los conocimientos generados en un proceso de aprendizaje, a la vez que facilitan la búsqueda de conocimientos generados por otras personas y fortalecen el intercambio de información y de experiencias. De acuerdo con Larose et al. (1999), la integración de las TIC en los procesos educativos debe ser el resultado de una visión integral del conocimiento, que refleje una construcción social de la realidad.

El elemento fundamental del tecno-constructivismo radica en considerar que el conocimiento es una construcción que se realiza mediante la interacción con diferentes recursos del ambiente de aprendizaje. Cabe mencionar específicamente las personas y los objetos, tales como las computadoras y sus programas, los cuales ofrecen simulaciones, juegos, bancos de datos, tutoriales y cursos, entre otros.

De ahí que se confirma la idea de Vygotsky (1995), según la cual el pensamiento es una construcción sociocultural y responde al contexto histórico en el que se desenvuelven los individuos. En relación con lo anterior, Papert (1997), incluye la Internet como un medio producido cultural para el aprendizaje, ya que es un invento reciente en el mundo tecnológico que ha transformado los espacios de relación y de comunicación entre personas y espacios.

\section{Comunidad Virtual de Aprendizaje (CVA)}

La Comunidad Virtual de Aprendizaje (CVA) es un medio pedagógico para vincular las TIC a los espacios educativos actuales, con el propósito de generar un diálogo efectivo con los participantes y entre los participantes, propiciar el aprendizaje activo y la experiencia colaborativa de pensar con otros. Lo anterior es factible si se parte del supuesto pedagógico de la interacción social como base de los procesos educativos, es decir, la comunicación es el eje central en la gestión de estas comunidades.

Estas comunidades pueden brindar experiencias significativas en la medida en que los entornos de aprendizaje virtual se perciban como personalizados por el usuario, se promueva la discusión y la guía en los procesos de intercambio en la comunidad de aprendizaje, así como la planificación. Es importante compartir las expectativas y los objetivos en la comunidad.

El concepto de CVA que se utilizó fue el de un grupo de personas con necesidades cognitivas y culturales (afines o no), que pueden encontrarse en el ciberespacio, quienes, a 
partir de una dinámica de trabajo colaborativo, construyen conocimiento interactuando en un lugar electrónico, extendiendo las posibilidades de interacción más allá de los límites geográficos. Su orientación es temática y funcional, pues su naturaleza se expresa a través de una plataforma, donde los participante aprenden en distintos espacios y tiempos diferentes, con aprendizaje colaborativo e individual, dentro de un ambiente de interacción y construcción.

La CVAA permitirá la participación del profesorado de las cuatro universidades públicas en todas las sedes del país, no sólo como sujetos del proceso de formación continua, sino como constructores de insumos teóricos y prácticos, que serán utilizados en la tarea de formar profesionales ambientalmente responsables. De esta forma, se contribuirá con la actualización en las diversas temáticas ambientales, se compartirán experiencias generadas y se crearán nuevos entornos de aprendizaje y colaboración.

En la figura 2, se presenta el modelo que seguirá la CVAA para lograr que el personal de las universidades estatales de Costa Rica, logre su formación ambiental en un entorno virtual de aprendizaje. Se observa como la autoformación virtual sobre aspectos ambientales contribuirá con el desarrollo de acciones para lograr la ambientalización curricular y generar nuevos proyectos universitarios en esta temática. 
FIGURA No. 2

Modelo

\section{AMBIENTALIZACION CURRICULAR}

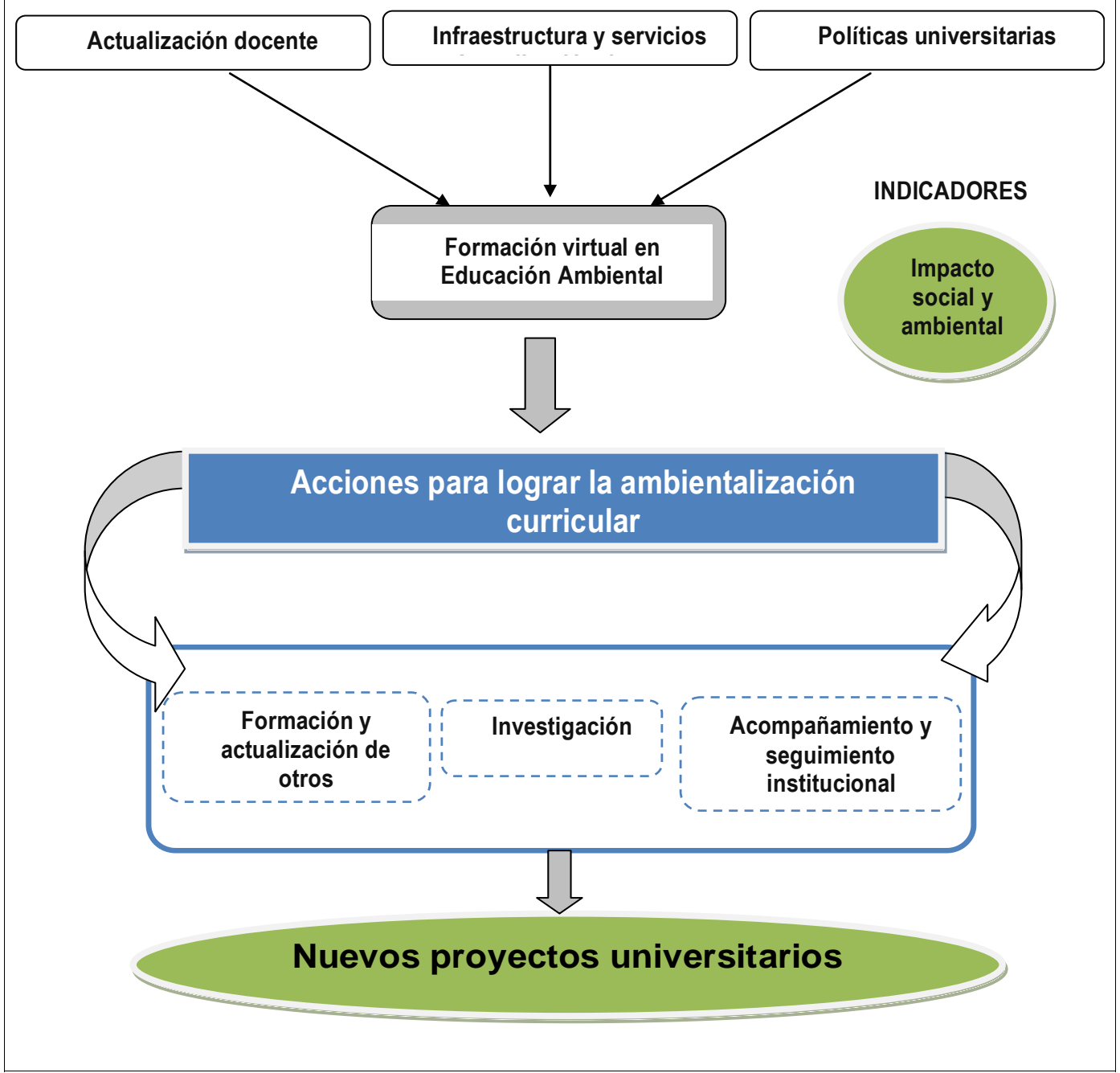

Fuente: Elaboración propia. 


\section{Comunicación Ambiental}

La comunicación ambiental permite captar la atención de las personas sobre los problemas ambientales actuales y eventuales, así como resaltar las potencialidades de un manejo adecuado del ambiente.

Sin embargo, la problemática ambiental es muy compleja e incluye temas sociales y económicos, por lo que es difícil lograr en forma rápida cambios de conciencia de las personas, lo que se debe reflejar en la comunicación ambiental, la cual debe formar una conciencia ambiental, generar conocimientos para facilitar la comprensión de los problemas ambientales, crear una responsabilidad crítica y fomentar actitudes basadas en valores para la protección y mejoramiento del entorno, así como impulsar aptitudes para la resolución de los problemas, la participación en la búsqueda de soluciones y de evaluación para lograr un desarrollo sostenible (Solano, 2001).

\section{DISEÑO DE LA COMUNIDAD VIRTUAL DE APRENDIZAJE AMBIENTAL (CVAA)}

La CVAA ha sido producto de una investigación que abarcó dos momentos: el diseño de la comunidad y su puesta en práctica. Por esta razón se fundamentó en el enfoque de la investigación -acción, que se caracteriza por integrar diferentes perspectivas teóricas y metodológicas para la gestión de procesos de cambio personales, organizativos e institucionales.

Así la CVAA se ha convertido en un espacio de interacción de enseñanza y aprendizaje donde los conocimientos se comparten y las experiencias enriquecen el quehacer universitario de manera integral y, además de motivar, socializar y lograr el intercambio sobre este eje transversal, ha permitido fortalecer la trascendencia de este recurso tecnológico para mover a toda la comunidad estatal universitaria a trabajar en la formación integral de sus estudiantes en la construcción de la nueva cultura ambiental.

\section{Etapas del proceso}

La construcción de la CVAA se planteó mediante la estructuración de etapas, las cuales permitieron incorporar la DA en la práctica del quehacer universitario. Estas etapas se desarrollaron en forma de espiral, donde la información obtenida en cada fase originó 
modificaciones y elecciones respecto a la siguiente y, por ende, al desarrollo global del proceso de investigación y de la construcción de la CVAA. Dichas etapas fueron las siguientes:

Diagnóstico: Se determinaron las condiciones básicas de recursos tecnológicos disponibles en las cuatro universidades estatales que participaron en el proyecto.

Diseño de la comunidad: Se realizó interdisciplinariamente y bajo los enfoques definidos: tecno-constructivismo, comunidad virtual de aprendizaje, Educación Ambiental, comunicación ambiental y Dimensión Ambiental.

Desarrollo de la comunidad: Se logró a partir de la elección de actividades en la Comunidad virtual, entre ellas la puesta en práctica de un curso de inducción.

Proceso de evaluación integrado. La investigación-acción permitió integrar los procesos de seguimiento y evaluación acorde con las acciones que se gestaron en el marco del desarrollo de la CVAA. Para ello, se propusieron criterios cuantitativos como: cantidad de personas que participan por componente, número de experiencias aportadas por la comunidad virtual, cuantificación de componentes realizados en la comunidad virtual y nivel de éxito o aprovechamiento. $\mathrm{Y}$ también aspectos cualitativos, como: las lecciones aprendidas de la experiencia, la percepción de los integrantes de la comunidad virtual sobre su propia transformación, el grado de consolidación de la comunidad y la presencia de la DA en el quehacer de los miembros de la comunidad.

Divulgación de la experiencia: se realizó mediante sesiones bimodales, es decir, tanto presenciales como virtuales en distintos espacios académicos de las universidades participantes. Se divulga por medio del sitio Web de la CVAA (www.comunidad.inie.ucr.ac.cr)

\section{Modalidades y escenarios para el aprendizaje}

La CVAA permitió implementar una visión tecno-constructivista, la cual se ha plasmado en la oportunidad de retroalimentar continuamente la bimodalidad como modelo de aprendizaje. De esta manera, se ha logrado que la formación continua del personal universitario se realice también mediante intercambios asincrónicos, no exclusivamente presenciales, lo que favorece el aprendizaje autónomo, cooperativo y colaborativo que enriquece el trabajo individual y grupal, evidenciándose el desarrollo de una red de conocimiento social.

Se organizó en al menos tres escenarios para el aprendizaje, a saber: 
- Presencial: sesiones programadas para el encuentro inicial y el cierre del curso de Inducción a la Comunidad Virtual de Aprendizaje Ambiental. Se propició un intercambio centrado en el diálogo y en el conocimiento de las experiencias previas de los participantes. En la sesión inicial se hizo una inducción acerca del curso y del uso de la plataforma de la comunidad. Al finalizar se compartieron las propuestas desarrolladas para dicho fin.

- Virtual: Actividades programadas para ser realizadas utilizando la plataforma www.comunidad.inie.ucr.ac.cr, tales como: grupos de discusión, debates, envío de materiales, devolución de las tareas y evaluaciones. Cada participante recibió información básica sobre el uso de la plataforma, los pasos para la inscripción, el programa y una actividad de incentivación para el inicio del curso. En la plataforma se ubicó un calendario con la información general de las tareas; también se facilitó el acceso a los materiales didácticos y se utilizó la herramienta para el intercambio de propuestas y su respectiva realimentación. Finalmente, se llevaron a cabo foros, para compartir en forma grupal experiencias, conocimientos, perspectivas sobre los desafíos y posibilidades para incluir la DA, así como de las propuestas que desarrollarían durante el curso. Cada participante planteó una propuesta afín con su quehacer universitario, de forma individual o grupal, ya que se matricularon personas de los sectores académico, administrativo y estudiantil.

- Individual: lecturas, el desarrollo de una propuesta para incorporar la DA, los materiales de apoyo y otros recursos para promover acciones a favor del ambiente en el quehacer universitario.

Estos escenarios de aprendizaje propiciados por las TIC incorporan los cambios de las relaciones espacio-temporales, y los objetivos educativos en el contexto sociocultural, lo cual se evidencia en el hecho de trasladar la experiencia de aprendizaje a otros espacios: el trabajo, el hogar, la educación y la diversión, así como centro de recursos de aprendizaje multimedia, entre otros.

\section{Estructura del sitio Web}

En el Cuadro No. 2 se presenta el esquema de la estructura del sitio Web de la Comunidad Virtual de Aprendizaje Ambiental (CVAA). 


\section{CUADRO No. 2}

Estructura del sitio Web de la CVAA

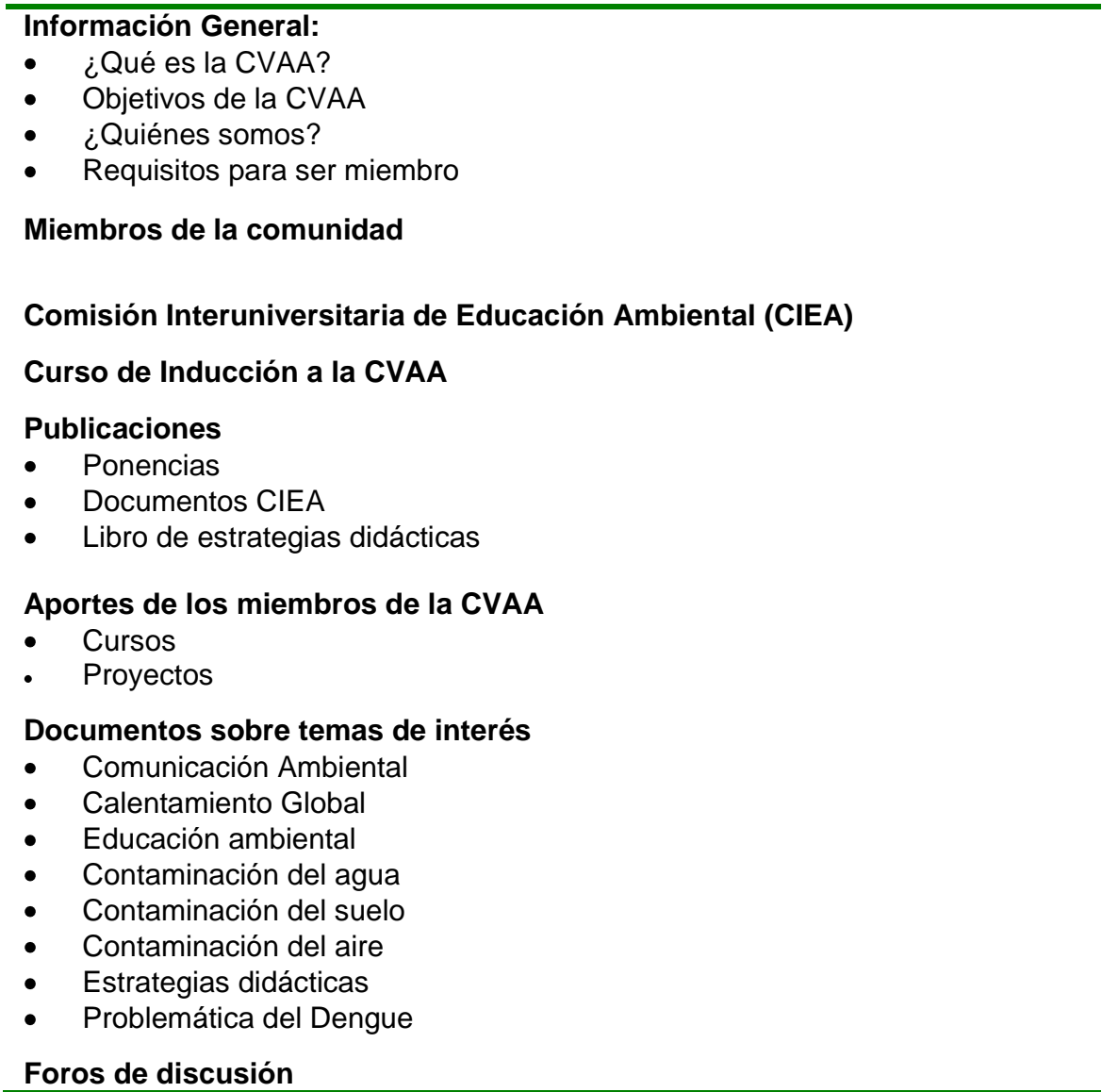

\section{Diseño del Curso de "Inducción a la Comunidad Virtual de Aprendizaje} Ambiental”

La experiencia de la CVAA derivó en el planteamiento del Curso de Inducción para incorporar la DA en el quehacer universitario. El diseño que se presenta, fue resultado del enriquecimiento y valoración que se produjo de experiencias realizadas con personal universitario y las lecciones de aprendizaje de las facilitadoras.

Está estructurado en una etapa previa de inscripción e información general, dos foros y tres módulos:

1. Acercamiento a la integración curricular de la Dimensión Ambiental.

2. Incorporación de la Dimensión Ambiental en mi quehacer.

3. Trascendiendo de la sensibilización a la acción. 
Por medio de este espacio los miembros de la comunidad se expresaron sobre las estrategias personales y grupales que aplicarían para trascender de la etapa de conocimiento y sensibilización sobre la problemática ambiental, a la acción en pro del entorno.

Finalmente los participantes compartieron mediante una presentación oral y escrita la propuesta de proyecto o curso, en la cual incorporaron la Dimensión Ambiental. Para ello se diseñó una guía que se presenta en el Cuadro No.3.

Una vez que cada participante realizó el análisis de su experiencia y escogió el tipo de estrategia para implementar la DA desde el contexto de su quehacer, se sugirió considerar los siguientes aspectos para que la propuesta fuera operativa. En el caso de docentes, se recomendó utilizar como guía el Cuadro No.3 partiendo de los siguientes aspectos:

- El perfil de sus estudiantes.

- El conocimiento que elaboran los estudiantes mediante el estudio de las teorías y contenidos que incluye su curso.

- Las estrategias para alcanzar esta formación de manera pertinente

- La manera en que evaluará esta formación

- Las fortalezas, las limitaciones y las expectativas que su curso ofrece para esta formación.

\title{
CUADRO No. 3
}

Guía para orientar la incorporación de la Dimensión Ambiental en los cursos de los participantes. CVAA 2009.

\begin{abstract}
I. Pasos recomendados para la implementación de la DA en un curso:
Dado que la integración de la Educación Ambiental como una dimensión en el currículo requiere una innovación en varios aspectos (Novo, 1991; Novo, 1993), éstos se plantearon con el propósito de que los utilice de guía para el análisis de la propuesta a generar en el curso, a saber:
\end{abstract}

- Conceptual: incorporación de contenidos relacionados con la temática ambiental.

- Metodológico: uso del entorno como centro de interés para estimular en sus estudiantes la observación, la experimentación y el análisis.

- Interdisciplinar: como método que favorece una forma de conocimiento articulado que permite integrar, no sumar, distintos enfoques científicos y tecnológicos para la interpretación de un problema ambiental.

- Transdisciplinar: como una forma de conocimiento, de pensamiento integrado, que puede llegar a generar modelos interdisciplinarios de trabajo en equipo y que actúen como un eje transversal en todo el proceso de formación estudiantil. 
- Proyectos integrales: participación activa de estudiantes para resolver problemas en un medio real.

- Unidades integradoras de enseñanza aprendizaje: módulos educativos estructurados con objetivos, actividades, sugerencias metodológicas y pautas de evaluación.

- Reestructuración del currículo: el ideal es llegar a un diseño curricular de carácter interdisciplinario.

Fuente: Elaboración propia.

Para quienes escogieron desarrollar un proyecto que conllevara la incorporación de la DA en su quehacer, se facilitó la guía que se presenta en el Cuadro No.4.

CUADRO No. 4

Guía para orientar la incorporación de la Dimensión Ambiental en proyectos. CVAA 2009.

\section{Pasos recomendados para la implementación en un proyecto}

Tal y como se señaló anteriormente, la educación ambiental, incluida como una dimensión, brinda experiencias de aprendizaje que permiten observar y analizar las relaciones de los seres vivos con el entorno e identificar los problemas que se originan de esa interacción. El diseño e implementación de un proyecto, permite desde la identificación de la problemática ambiental hasta la participación activa en la ejecución de posibles soluciones a los problemas identificados, para ello, se plantearon con los siguientes pasos:

1. Problemática ambiental a abordar: implica investigar, ordenar, clasificar y sistematizar información sobre el problema ambiental a abordar. Es importante además, identificar y plantear soluciones viables al problema.

2. Qué quiere lograr: con base en la información obtenida en el paso 1, planteé los objetivos del proyecto a realizar para contribuir con la minimización del problema ambiental seleccionado.

3. Para qué: justifique brevemente por qué y para qué desarrollará el proyecto.

4. Cómo y dónde: defina la metodología que empleará para desarrollar las actividades del proyecto.

5. Para quién (es): defina el grupo al que va dirigido el proyecto.

6. Con qué y qué necesita para lograrlo: elabore una lista de los recursos humanos y financieros que requiere para desarrollar las actividades.

7. Qué esperan lograr: precise los resultados o productos esperados, así como la forma en que se evaluarán.

Fuente: Elaboración propia 
De igual modo, en el curso de inducción a la CVAA se utilizaron dos instrumentos para valorar los alcances de las actividades propuestas. Se realizó un cuestionario sobre conocimientos y experiencias previas de los participantes relacionadas con ambiente, para ser aplicado con la técnica de pre y post prueba. Además, se elaboró otro cuestionario que permitió realimentar específicamente la experiencia desarrollada durante el curso. Asimismo, se tomaron en cuenta los aspectos técnicos del uso y acceso a la plataforma, así como las temáticas indicadas para integrar la DA en el quehacer universitario.

\section{RESULTADOS OBTENIDOS \\ Construcción de la CVAA}

Como parte de la dinámica de aprendizaje en el curso de inducción a la CVAA y dada la importancia que tiene para el funcionamiento de la misma, se consideró oportuno valorar esta experiencia, con el modelo de Salmon-Brenson (Brenson-Lazan, s.f.), que recomienda cinco etapas para la construcción de una comunidad virtual de aprendizaje, que son: motivación, socialización, intercambio, construcción y trascendencia.

1. Motivación. Por medio de este curso se determinó que los participantes son académicos, funcionarios administrativos y estudiantes universitarios comprometidos con el reto de incorporar la DA en su quehacer. La revisión de los avances y propuestas elaboradas en el curso, mostraron que las personas que terminaron el curso, están deseosas de asumir dicho desafío.

2. Socialización. Por medio del primer foro y la presentación inicial de los participantes en el curso, se dieron a conocer detalles de su quehacer académico e intereses en la temática ambiental. Creo que el segundo foro también permitió socializar al igual que otros espacios de la CVAA

3. Intercambio. El curso permitió el intercambio de experiencias. Los integrantes presentaron propuestas de proyectos, y recibieron aportes de sus compañeros y de las facilitadoras, lo que permitió que ampliaran su aprendizaje hacia otras actividades, estrategias y proyectos, para la inclusión de la DA en su trabajo cotidiano.

4. Construcción. Para construir esta comunidad virtual que pretende contribuir con la formación integral de los futuros profesionales, es fundamental que los participantes del curso, se conviertan en multiplicadores y promotores de este reto universitario. 
Por lo anterior, en el sitio electrónico de la CVAA se incluyó un espacio, que permitirá conocer y compartir las propuestas de los proyectos y cursos en los que se plantea incluir la DA, para que sirvan de modelo a otros universitarios.

5. Trascendencia. Se espera que este curso así como los espacios y productos que ofrece la CVAA se conviertan en el motor que active a toda la comunidad estatal universitaria a trabajar en la formación integral de los estudiantes y en la construcción de la nueva cultura ambiental. Los participantes en los cursos de inducción y las facilitadoras, consideran importante informar e involucrar a las autoridades universitarias pertinentes, para que apoyen los diferentes trabajos que se proponen para lograr este reto.

En la Figura No. 3 el sitio web de la CVAA se convertirá en un instrumento primordial para contribuir a la reflexión y a la gestión de experiencias similares para incorporar la Dimensión Ambiental en el quehacer universitario (García et al., 2011)

\section{FIGURA No. 3}

Portada del sitio Web de la Comunidad Virtual de Aprendizaje Ambiental

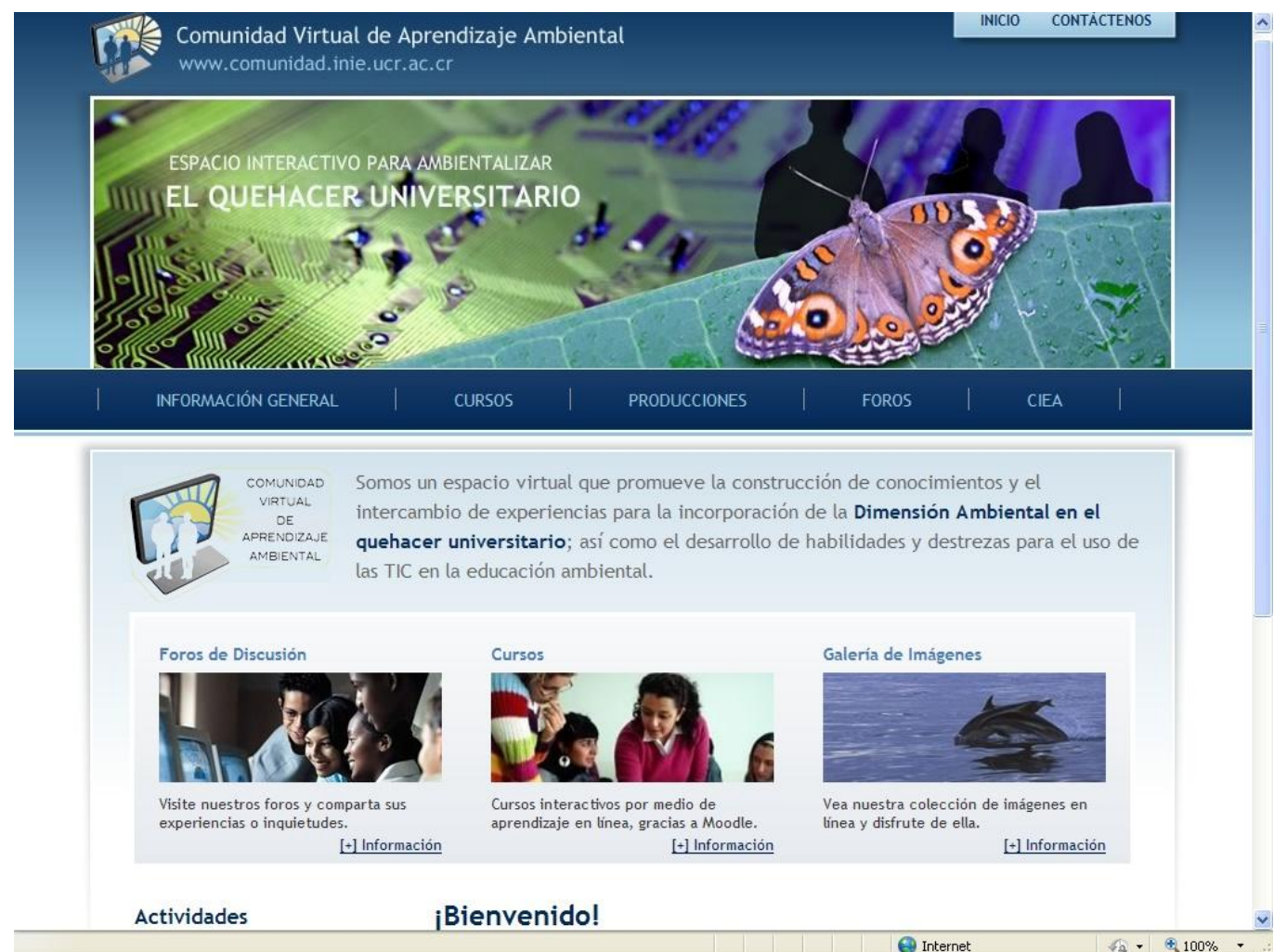




\section{Productos desarrollados por los participantes en el Curso de Inducción a la CVAA}

Se integró la Dimensión Ambiental en 33 cursos de diferentes disciplinas que se implementaron en las cuatro universidades estatales y se enriqueció en 28 proyectos de gestión ambiental (Cuadro No. 5). En algunas capacitaciones, los participantes sólo presentaron proyectos.

\section{CUADRO 5.}

Materias en que se incorporó la Dimensión Ambiental como eje transversal

\begin{tabular}{|c|c|}
\hline \multicolumn{2}{|c|}{ CURSO: OCTUBRE-NOVIEMBRE 2007} \\
\hline $\begin{array}{l}\text {-Experiencia profesional I en Educ. Preescolar } \\
\text {-Fundamentos de Didáctica }\end{array}$ & $\begin{array}{l}\text {-Física General I } \\
\text {-Introducción a la problemática ambiental }\end{array}$ \\
\hline -Economía Ambiental & -Práctica Docente en Educación Primaria \\
\hline -Teoría de la Educación & -Ecuaciones diferenciales \\
\hline \multicolumn{2}{|c|}{ CURSO: ENERO- FEBRERO 2008} \\
\hline $\begin{array}{l}\text {-Ambiente Humano } \\
\text {-Agro-estadística I } \\
\text {-Agromática I } \\
\text {-Inglés I para Administración } \\
\text {-Protección de Cultivos I } \\
\text {-Administración de Recursos Humanos I. }\end{array}$ & $\begin{array}{l}\text {-Introducción a la Ciencia, Técnica y -Tecnología } \\
\text {-Edafología } \\
\text {-Protección de Cultivos II } \\
\text {-Actividad Deportiva I } \\
\text {-Administración de Recursos Humanos II. } \\
\text {-Biología general }\end{array}$ \\
\hline \multicolumn{2}{|c|}{ CURSO: JULIO- SETIEMBRE DEL 2008} \\
\hline $\begin{array}{l}\text {-Relación Suelo Planta } \\
\text {-Planificación y Manejo del Paisaje con un } \\
\text { enfoque agroambiental } \\
\text {-Fundamentos de Didáctica } \\
\text {-Bases psicosociales de la Educación }\end{array}$ & $\begin{array}{l}\text {-Informática Aplicada a los Negocios } \\
\text {-Educación para la diversidad } \\
\text {-Principios de la medición y la evaluación } \\
\text {-Informática educativa }\end{array}$ \\
\hline \multicolumn{2}{|c|}{ CURSO: SETIEMBRE - OCTUBRE DE 2009} \\
\hline $\begin{array}{l}\text {-Diseño y propuesta para el contenido y } \\
\text { estrategia educativa de seminarios de realidad } \\
\text { nacional (SRN) y Trabajo comunal universitario } \\
\text { (TCU) }\end{array}$ & $\begin{array}{l}\text {-Problemática de la contaminación atmosférica y sónica } \\
\text {-Problemas ambientales especiales: Sistemas de Gestión ambiental }\end{array}$ \\
\hline \multicolumn{2}{|c|}{ CURSO: ABRIL-MAYO 2010} \\
\hline $\begin{array}{l}\text { UNA razón para cambiar...soy parte del } \\
\text { ambiente } \\
\text { Propuesta educativa ambiental PEA } \\
\text { Ambientalización del quehacer docente }\end{array}$ & $\begin{array}{l}\text { La dimensión ambiental en el quehacer del INEINA } \\
\text { Foro de discusión: Dimensión Ambiental en la UNA } \\
\text { Ambientando al CIDE }\end{array}$ \\
\hline \\
\hline \multicolumn{2}{|c|}{$\begin{array}{l}\text { Intencionalidad didáctica para la acción } \\
\text { ambiental comunal }\end{array}$} \\
\hline \multicolumn{2}{|c|}{ CURSO : MARZO - ABRIL 2011} \\
\hline $\begin{array}{l}\text { Incorporación de la Dimensión Ambiental en la } \\
\text { asignatura Desarrollo tecnológico y } \\
\text { sostenibilidad }\end{array}$ & Inclusión del componente ambiental en el curso de Hidrología \\
\hline
\end{tabular}

Fuente: Elaboración propia 


\section{CUADRO No. 6.}

Proyectos en que se incorporó la Dimensión Ambiental como eje transversal.

\section{CURSO: OCTUBRE-NOVIEMBRE 2007}

Centro de Acopio Institucional

Salvando nuestra familia : La Dimensión Ambiental como un valor en nuestras vidas

Material multimedia para incentivar la incorporación del eje ambiental

Incorporación de la Dimensión Ambiental en el Proyecto Institucional de Manejo de Desechos

\section{CURSO: ENERO- FEBRERO 2008}

Incorporación del ambiente como eje transversal en los currículos del ITCR desde el Departamento de Vida Estudiantil y Servicios Académicos

Incorporación del ambiente como eje transversal en los currículos del ITCR desde la Unidad de vigilancia.

\section{CURSO: JULIO- SETIEMBRE DEL 2008}

Concienzar sobre el ahorro en agua y electricidad y el reciclaje de papel y cartón en el Centro Universitario UNED. Turrialba.

Proyecto de Reutilización del Papel Blanco y Periódico UNED. Turrialba

\section{CURSO: SETIEMBRE - OCTUBRE DE 2009}

Innovación educativa en gestión integrada de la salud y el ambiente

Problemática ambiental de la cuenca Arenal-Tempisque

Campaña de Divulgación del Programa de Educación Ambiental del Proceso Participativo para el Manejo Integral de la Quebrada Los Negritos a la Comunidad Universitaria

Sistema de Reconocimiento del desempeño ambiental para unidades de la Ciudad Universitaria Rodrigo Facio

Capacitación y aprendizaje de la población de la subcuenca del río Purires.

Campaña de Información a la comunidad de Tirrases sobre el Cierre Técnico del Relleno

Sanitarios Río Azul

\section{CURSO: 2011}

Propuesta para minimizar el impacto ambiental en Administración de Empresas, ITCR

Cuidando los recursos de papel y tinta en la Escuela de Ciencias Sociales, ITCR

Ahorro energético. Oficina de Auditoría Interna, ITCR

Grupo social: cápsulas sobre el medio ambiente

Desarrollo de un Programa de compras verdes para el Instituto Tecnológico de Costa Rica

Estrategia de las Cuatro Erres

Ahorro de agua/ Unidad Tesorería ITCR

Fuente: Elaboración propia 


\section{CONCLUSIONES}

La Comunidad Virtual de Aprendizaje Ambiental se ha consolidado como un recurso muy valioso para facilitar el intercambio de conocimientos, el análisis y la reflexión de los diversos sectores participantes, en el proceso de incorporación de la DA en los diversas actividades universitarias, facilitando el desarrollo de estrategias en beneficio del ambiente.

Este espacio de educación virtual permitió además, el trabajo tanto individual como grupal, rompiendo con las barreras que imponen la distancia, como el caso de los cursos en San Carlos y Turrialba y las múltiples ocupaciones de todos los involucrados, ofreciendo de esta manera, un recurso accesible a la disponibilidad horaria de cada uno. Tal y como lo señalan Mas y colaboradores (2006), este medio permite una activa interacción, aprendizaje colaborativo, intercambio de recursos entre los participantes y entre las facilitadoras, permitiendo una evaluación constructiva y un significado socialmente construido.

Además la experiencia obtenida mediante el diseño del curso de inducción a la CVAA mostró que el uso de plataformas es una manera de compartir los aprendizajes construidos, para facilitar situaciones en las que se incorpora la DA como un eje transversal, tal es el caso de los cursos y proyectos pertenecientes a diferentes instancias de la vida universitaria, esto a su vez generó sensibilización, conocimientos y destrezas para incorporar la DA en el quehacer de los participantes, promoviendo el compromiso y la acción ambiental.

De igual manera, es importante reconocer a la CVAA en un espacio interactivo útil para los sectores académico, administrativo y estudiantil de las universidades estatales costarricenses. Para mantenerlo vigente es importante fortalecer las estrategias que garanticen su óptimo funcionamiento, sostenibilidad y renovación, con miras al cumplimiento de su propósito de creación, a saber, constituirse en un espacio educativo virtual que promueva en dichos sectores la construcción de conocimientos, habilidades y destrezas y generador de acciones a favor del ambiente.

Y está acorde con el cumplimiento de las políticas ambientales de las cuatro universidades estatales costarricenses y con lo que mencionan Zúñiga y Arnáez (2010), que señalan la importancia de las CVA para desarrollar el proceso de enseñanza aprendizaje en la sociedad actual donde la reflexión, la discusión y el intercambio producen experiencias significativas y se convierten en sitios para realizar trabajos y actividades en conjunto como proyectos de investigación, extensión, acción social y los programas de formación interuniversitaria. 


\section{Referencias}

Brenson-Lazan. Gilbert (2001). Etapas de desarrollo y facilitación en una comunidad virtual de aprendizaje. Amauta Internacional. LLC. Recuperado el 15 de marzo de 2006, de http://www.amauta.org/DesarrolloComunidadVirtual.pdf

Cabero Almenara, Julio. (2006). Comunidades virtuales para el aprendizaje. Su utilización en la enseñanza. Edutec. Revista Electrónica de Tecnología Educativa, (20), 1-34. Disponible en http://edutec.rediris.es/Revelec2/revelec20/cabero20.pdf

Cabello Fernández, Florencio; Leiva Olivencia, Juan; Rascón Gómez; María Teresa. (2006). Comunidades de aprendizaje y educación digital: Reflexiones desde una perspectiva pedagógica de la comunicación. Grupo de trabajo: Comunicación y educación. IX Congreso IBERCOM. Sevilla-Cádiz, España. 2006. Consultado el 7 de setiembre de 2008, de www.hapaxmedia.net/ibercom/cabelloFernandezDelgadoFlorencio.php

Caduto, Michael. (1985). A guide for environmental values. UNESCO-UNEP International. Environmental Education Program (Environmental Education Serie 13). France: UNESCO.

Curiel Ballesteros, Arturo. (1997). Educación ambiental: evolución de un concepto. Educación Ambiental de Latinoamérica. Órgano Informativo de Educadores Ambientales. (Especial), 9-10.

Elliott, John. (1996). El cambio educativo desde la investigación acción. Madrid: Ediciones Morata.

Emmons, Katherine. (1997). Perspectives on environmental acting: reflection and revision though practical experience. Journal of Environmental Education, 29(1), 34-44.

García Fallas, Jacqueline; Hernández Rojas, Lidia M.; Zúñiga Vega, Claudia; Arnáez Serrano, Elizabeth.; Charpentier Esquivel, Claudia; Carrillo Delgado, María de los Ángeles. (2011). Comunidad Virtual de Aprendizaje: Un espacio para la formación ambiental. San José, C.R.: INIE.

Hernández Rojas, Lidia; García, Fallas; Jacqueline. (2009). Hacia la construcción de una cultura ambiental universitaria. 15 años de labor de la CIEA. San José, C.R.: Consejo Nacional de Rectores.

Larose, François; David, Robert; Dirand, Jean Marie; Lenoir, Yves; Roy, Grenon. (1999). Rapport de recherche portant sur le profil d'utilisation des TIC en pédagogie universitaire à Sherbrooke. Sherbrooke: Université de Sherbrooke, Vice-rectorat à l'enseignement. Disponible en: http://www.usherb.ca/PP/documents/tic99

Mas, O.; Jurado, P.; Ruiz, C.; Ferrández, E.; Navío, A.; Sanahuja, J.M.; Tejada, J. (2006). Las comunidades virtuales de aprendizaje. Nuevas fórmulas, viejos retos en los procesos educativos. PixelBit, (35), 1462-1466. 
Novo, María. (1991). Educación ambiental ( $2^{\circ}$ ed.). Bogotá: Red Editorial Martínez Roca.

Novo, María. (1993). La Educación Ambiental en la universidad. En: Educación Ambiental y Universidad. Congreso iberoamericano de Educación Ambiental. Una estrategia al porvenir. Coordinación General de Ecología y Educación Ambiental, Universidad de Guadalajara, México.

Papert, Seymour. (1997). La familia conectada: padres, hijos y computadoras. Buenos Aires: Emecé.

Pazos, María; Pérez, Adolfina; Salinas, Jesús. (2001). Comunidades Virtuales: de las listas de discusión a las comunidades de aprendizaje. Recuperado el 5 de febrero de 2008, de

www.imacmexico.org/file download.php?location $=S$ U\&filename $=11235195271 \mathrm{CVIRTU}$ ALES01.pdf

Solano, David. (2001). Comunicación y generación de conciencia ambiental. Tópicos en Educación Ambiental, 3 (7), 52-57.

Vygotsky, Lev. (1995). Pensamiento y Lenguaje. Barcelona: Paidós.

Zúñiga Vega, Claudia, Arnáez Serrano, Elizabeth. (2010). Comunidades virtuales de aprendizaje, espacios dinámicos para enfrentar el SXXI. Tecnología en marcha, 23 (1), 19-28. 